\title{
Oportunidades perdidas en la enseñanza de especialidades médicas, a propósito de la influenza y COVID-19
}

\author{
Jesús Reyna-Figueroa, ${ }^{1 *}$ Arely A. Bejarano-Juvera, ${ }^{2}$ César A. Arce-Salinas, ${ }^{3}$ Hilario Martínez-Arredondo ${ }^{4}$ \\ y Rodolfo Lehmann-Mendoza \\ ${ }^{1}$ Departamento de Enseñanza e Investigación; ${ }^{2}$ Servicio de Pediatría; ${ }^{3}$ Dirección; ${ }^{4}$ Gerencia de Prevención Médica; ${ }^{5}$ Subdirección de los Servicios
} de Salud. Petróleos Mexicanos, Hospital Central Sur de Alta Especialidad, Ciudad de México, México

\section{Resumen}

La enseñanza en las especialidades médicas es un rubro de los sistemas de salud que merece consideraciones especiales de acuerdo con las lecciones aprendidas de la influenza y COVID-19. Deben reevaluarse los programas educativos y las estrategias de capacitación implementadas, ya que la preparación escolar de la mayoría de los alumnos no les permite ser considerados como personal que pueda enfrentar esos problemas globales. Los cursos de especialización han crecido en número, pero su principal amenaza es la suspensión de los programas académicos o que sean parcialmente cubiertos como consecuencia de no implementar estrategias operativas funcionales durante una contingencia.

PALABRAS CLAVE: COVID-19. Influenza. Pandemia. Educación médica. Especialidades médicas.

\section{Opportunities in the teaching of medical specialties, regarding influenza and COVID-19}

\begin{abstract}
Medical specialties' teaching is an area of health systems that deserves special consideration in light of the lessons learned from influenza and COVID-19; educational programs and implementation of the training strategies that are used must be reevaluated, since the level of training of most specialty students does not allow to consider them as personnel who can face these global problems. The number of specialization courses has exponentially grown, and their main threat is the cancellation or partial execution of their academic programs as a consequence of not implementing functional operational strategies during a contingency.
\end{abstract}

KEY WORDS: COVID-19. Influenza. Pandemic. Medical education. Medical specialties.

\section{Introducción}

Las pandemias infecciosas se producen cuando emerge una nueva cepa microbiana virulenta contra la cual la población humana no tiene inmunidad y causan un número importante de enfermos y muertes. En el siglo XX, el virus de la influenza ocasionó tres de ellas: la gripe española (1918), la gripe asiática (1957) y la gripe de Hong Kong (1968), ${ }^{1}$ y una más en 2009 provocada por el tipo A subtipo H1N1. ${ }^{2}$ Los Centros para el Control y la Prevención de Enfermedades notificaron al menos 52 millones de muertes acumuladas entre las cuatro, ${ }^{3,4}$ lo que corresponde aproximadamente a 18 millones de muertes menos que las acontecidas por las dos guerras mundiales, ${ }^{5,6} 118$ veces más que los 439 mil fallecimientos por los cinco terremotos con mayor número de defunciones de la historia moderna. COVID-19 acumula 9.8 veces más muertes
Correspondencia:

*Jesús Reyna-Figueroa

E-mail: jesusreynaf@gmail.com
Gac Med Mex. 2020;156:321-327

Disponible en PubMed

www.gacetamedicademexico.com CC BY-NC-ND (http://creativecommons.org/licenses/by-nc-nd/4.0/). 


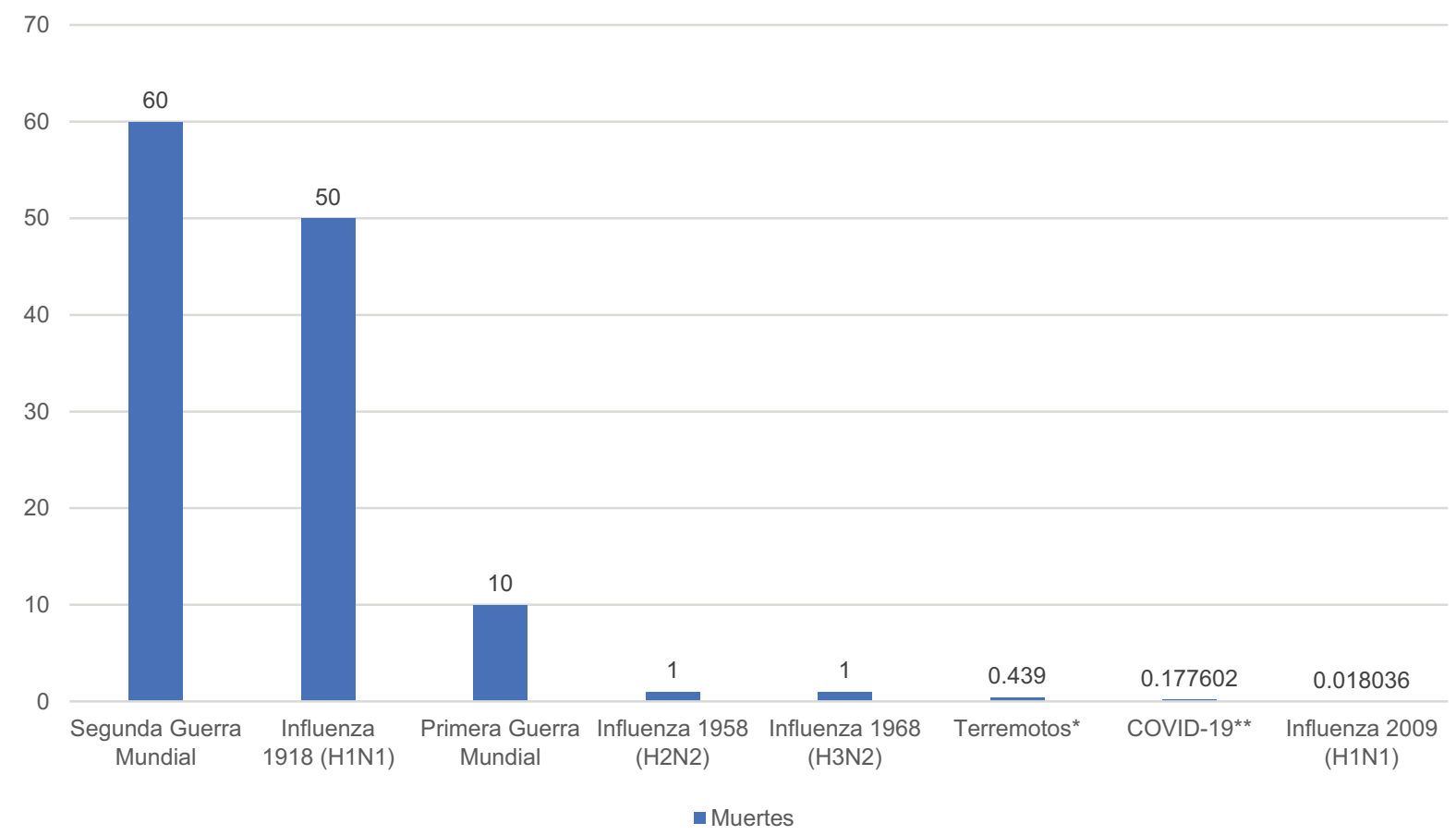

Figura 1. Número de muertes reportadas históricamente en pandemias y desastres. Escala $=n \times 1$ millón. *Suma de muertes de los cinco terremotos considerados más mortíferos: Haití en 2010, Perú en 1970, Chile en 1939, Guatemala en 1976 y Nicaragua en $1972 .{ }^{* *}$ Cifra de muertes por COVID-19 al 21 de abril de 2020.

que las causadas por la influenza H1N1 en 2009 (Figura 1).

Las crisis sanitarias son situaciones que proporcionan la oportunidad de reordenar los sistemas de salud, ya que para enfrentarlas se modifican las acciones que muestran limitaciones durante la contingencia. ${ }^{7}$ El consenso general establece que las pandemias son inevitables, por lo que podría suponerse que el mundo está preparado para la atención de estos eventos de gran impacto poblacional; 8,9 sin embargo, los analistas han reiterado que no hay una preparación suficiente para una respuesta adecuada y pronostican el colapso de los sistemas hospitalarios y la pérdida de muchas vidas por esa razón. ${ }^{10}$

Según los expertos, después de superar la crisis por COVID-19, los problemas en la economía, la educación y la salud serán las consecuencias para los países inadecuadamente preparados. ${ }^{11-13}$ Desafortunadamente, en medicina se desaprovechan las oportunidades que generan los cambios epidemiológicos, demográficos y económicos para realizar las adecuaciones a sus programas, con pérdidas de magnitud similar a ignorar las ocasiones de vacunar a los individuos. ${ }^{14-16}$

La educación médica es un rubro de los sistemas de salud que merecería consideraciones especiales durante el tiempo que coinciden los periodos pos $y$ prepandémicos (espacio que se inicia cuando se supera una crisis y se abre una nueva alerta en espera de otra contingencia), ${ }^{17}$ durante el cual se pueden analizar las lecciones aprendidas. En este periodo debe ser imperativo mejorar la enseñanza en los cursos de medicina, con base en el análisis de las acciones realizadas, evaluando los aciertos, poniendo énfasis especial en los errores y puntualizando las mejoras. Los resultados y las conclusiones serán las bases para la preparación prepandémica de los recursos humanos, la mejora en los planes de estudio, el aseguramiento de insumos y la elaboración de acuerdos interinstitucionales; siempre considerando que este periodo es de duración incierta ya que es difícil predecir cuándo será la próxima crisis sanitaria. En ambos periodos, que realmente son inseparables e indivisibles, el factor común siempre será la vigilancia epidemiológica y la mejora tecnológica (Figura 2).

\section{Oportunidades en la educación de especialidades médicas}

La enseñanza en salud, considerada en nuestros días como anacrónica, se encuentra en medio de una transformación, en la que deben reevaluarse los requisitos para ingresar a un curso de especialización, 


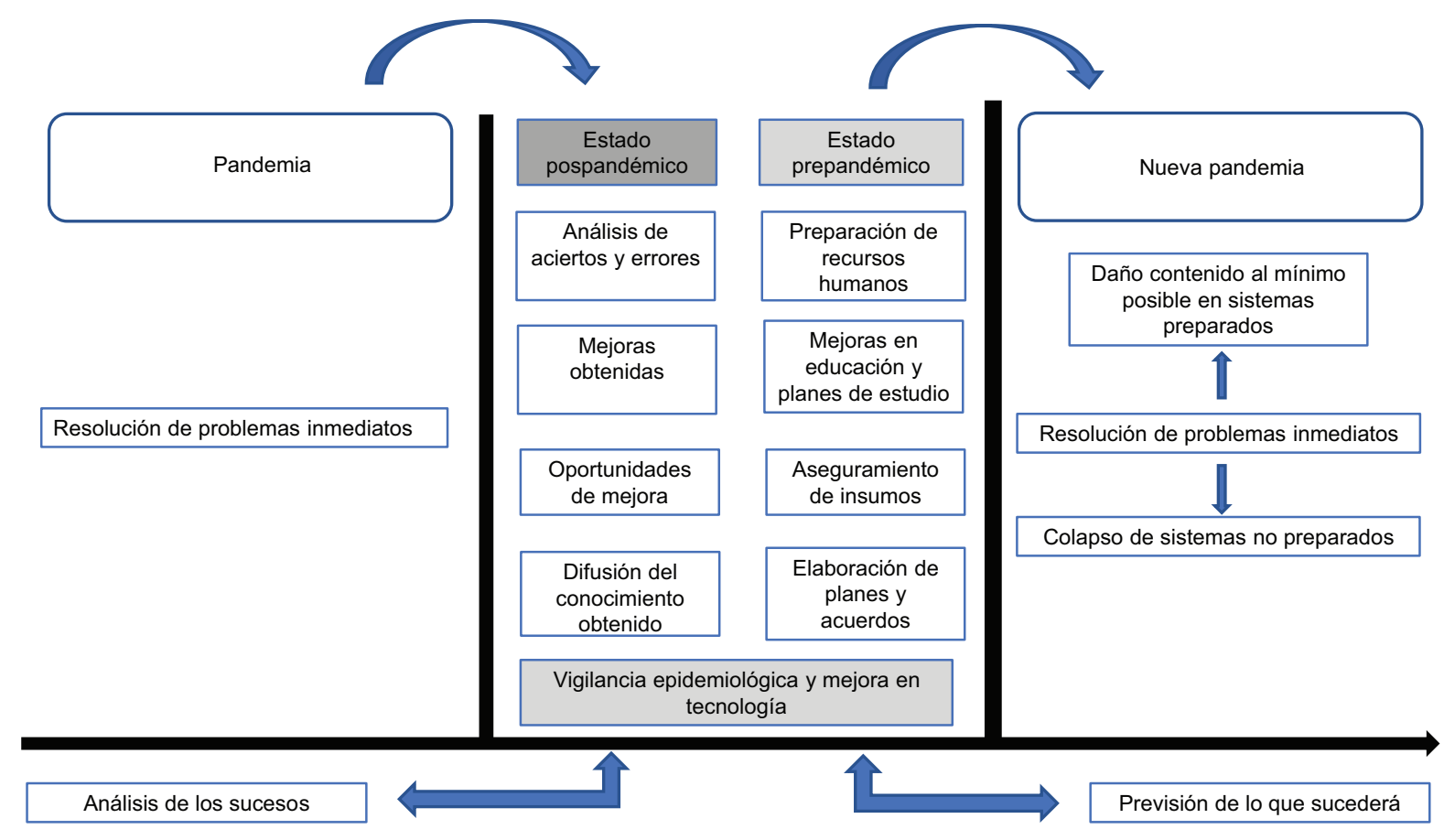

Figura 2. Los sistemas de salud desempeñan un papel de liderazgo en las pandemias y establecen acciones para la resolución de problemas inmediatos. Una vez que la pandemia se ha superado, se preparan las acciones para contener la siguiente. En el periodo en el que convergen ambos momentos, pos y prepandemia, existe la oportunidad de reestructurar la educación en las especialidades médicas, buscar estrategias para que en un futuro no sean interrumpidas y para mejorar el conocimiento del personal de salud.

la calidad de los programas, las estrategias de capacitación utilizadas y la manera como los recursos humanos pueden incluirse en el sistema que los formó, para que la inversión realizada retorne beneficios a la organización. ${ }^{17}$

Entonces, las crisis sanitarias tienen al menos dos vertientes de oportunidad para las especializaciones médicas. La primera puede modificar la forma como se enseña en las especializaciones médicas, de tal suerte que en eventos futuros las medidas de contención y mitigación no impacten en el desarrollo de los programas operativos y eviten su interrupción. Generalmente, las medidas para contener el contagio de microorganismos que se trasmiten de persona a persona, como los de índole respiratorio, se basan en el distanciamiento y aislamiento social, con los que se busca disminuir la velocidad de propagación de la enfermedad; $;, 18$ en un sistema mal preparado, estas medidas contribuyen a implementar cursos incompletos, con riesgo de la formación inadecuada de los futuros médicos especialistas.

La otra vertiente respecto a la educación médica está relacionada con la capacidad de las pandemias para revelar las respuestas deficientes de los sistemas, en las que destacan los científicos con limitado conocimiento y las dificultades en la toma de decisiones..$^{19,20}$
Los sistemas de salud en materia docente deben estar mejor preparados, disponer de trabajadores capacitados en el manejo de los protocolos generales de actuación; de no ser así, en conjunción con las escuelas de medicina deben adquirir una mayor responsabilidad en la preparación de los médicos que en el futuro enfrentarán las contingencias. ${ }^{21}$ Cada profesional de la salud debería poseer los conocimientos básicos que coadyuven a disminuir el caos, la resistencia y el pánico, pero, sobre todo, para que sea un verdadero apoyo en la ejecución de las decisiones, protegido por el enfoque global de un sistema que funciona independientemente de la naturaleza del patógeno o de su origen. ${ }^{10}$

\section{Factores asociados al cumplimiento de programas académicos durante una pandemia}

El número de cursos de especialización en las residencias médicas ha crecido exponencialmente. En forma contradictoria, la inversión y el aumento del presupuesto para soportar su éxito no parece ser una prioridad para los sistemas de salud, ${ }^{22}$ si bien es evidente que los países que más han invertido en educación médica y que poseen un sistema de selección 


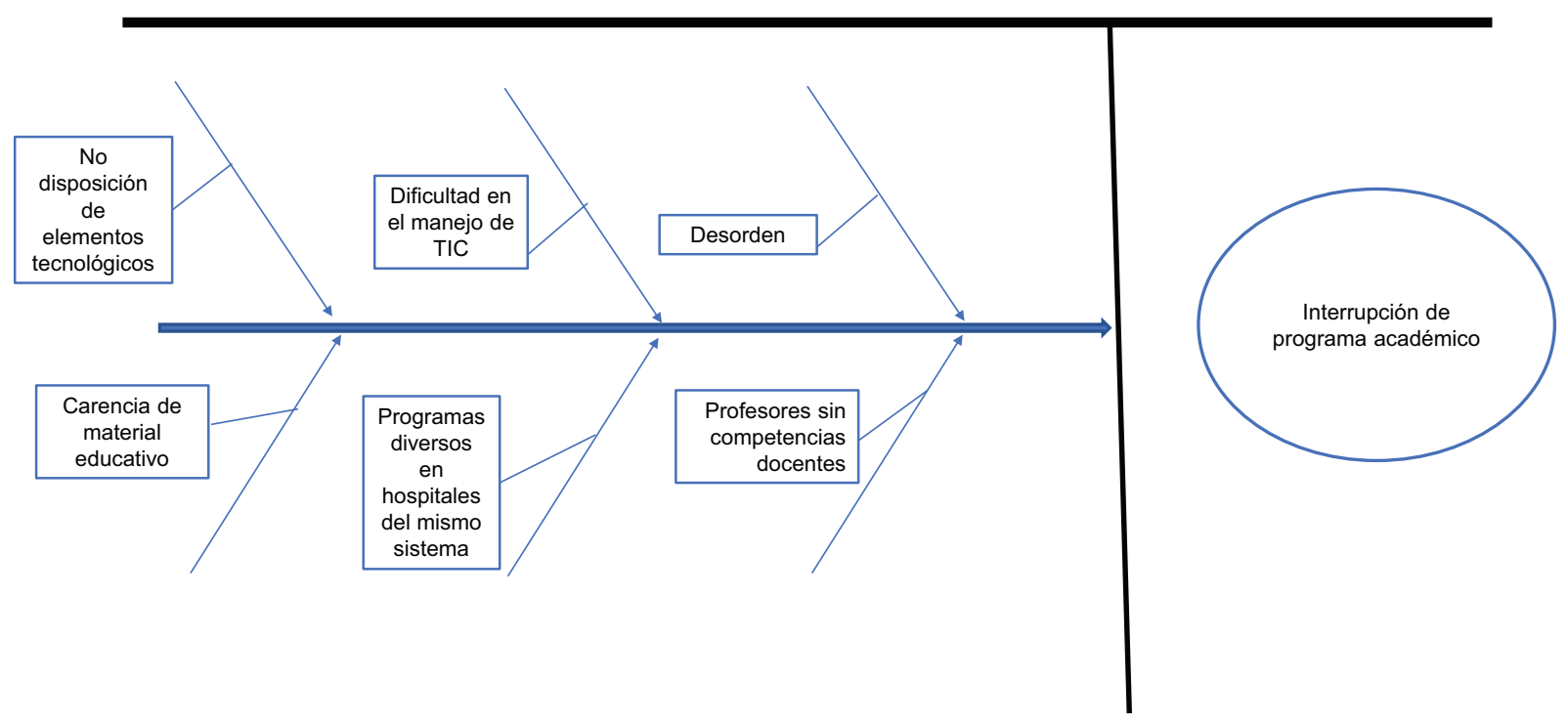

Figura 3. Factores asociados a la interrupción de programas académicos en los cursos de especialidades médicas durante una pandemia. TIC = tecnologías de la información y la comunicación.

estructurado han mejorado sus organizaciones hasta convertirse en los más importantes del planeta. ${ }^{23}$

Además de la urgencia y prioridades que se deben solventar durante una pandemia, ${ }^{24}$ los cursos hospitalarios de especialización médica tienen como principal amenaza la suspensión de sus programas académicos como consecuencia de su insuficiencia funcional y la falta de estrategias educativas que los doten de solvencia. ${ }^{25}$ El desorden administrativo, la improvisación, el desconocimiento o no disponibilidad de tecnologías de la información y las comunicación (TIC), la falta de bancos de información con material educativo o la no adquisición de competencias docentes por parte de los profesores constituyen las principales causas que favorecen que un programa académico no continúe en una contingencia (Figura 3). Lograr nuevos modelos docentes basados en el diseño educativo, el uso de tecnología emergente y el enfoque en las necesidades futuras de atención médica son los principales retos de cualquier institución inmersa en la educación que se considere exitosa. ${ }^{26}$

El uso de TIC para el aprendizaje en línea o a distancia puede solventar la imposibilidad de realizar sesiones con la técnica grupal tradicional durante los brotes de enfermedades de índole respiratorio. ${ }^{27}$ Son pocos los estudios que reportan la experiencia en el uso de TIC en las residencias médicas, si bien la idea general es que son muy utilizadas; sin embargo, se requiere mayor integración de los planes de estudio. ${ }^{28}$
Las transmisiones en línea en tiempo real o diferido son herramientas de gran utilidad no solo en crisis sanitarias; su empleo como complemento o como forma principal de contacto es cada vez más común en profesiones en las cuales el tiempo es limitado y se opta por revisar algún contenido en horarios preestablecidos. El aspecto económico representa un argumento también a favor: el aprendizaje en línea es considerado de bajo costo, por lo que podría aprovecharse más en medicina. ${ }^{29}$

A partir de estos conceptos, las estrategias colaborativas-cooperativas constituyen una opción de mejora, algunas basadas en "el aula invertida", donde cada alumno revisa los contenidos temáticos a su ritmo y preserva las reuniones grupales virtuales para realizar ejercicios o simulaciones que refuercen lo aprendido. Esta estrategia incluye la realización de ensayos y análisis de videos, así como la resolución virtual de problemas.

En las sesiones grupales se debe favorecer la formación de equipos para solucionar ejercicios problema, analizar textos, usar diarios de doble salida y concursar en juegos, de tal manera que se evite el esquema tradicional del maestro ante alumnos pasivos, quienes deberán responsabilizarse de las actividades; el trabajo en equipo los obliga a participar en las sesiones. Esta debería ser la forma rutinaria para trabajar la parte teórica de los cursos, exista o no una emergencia epidemiológica. Como complemento, las escuelas de medicina deben instituir programas para elevar la 


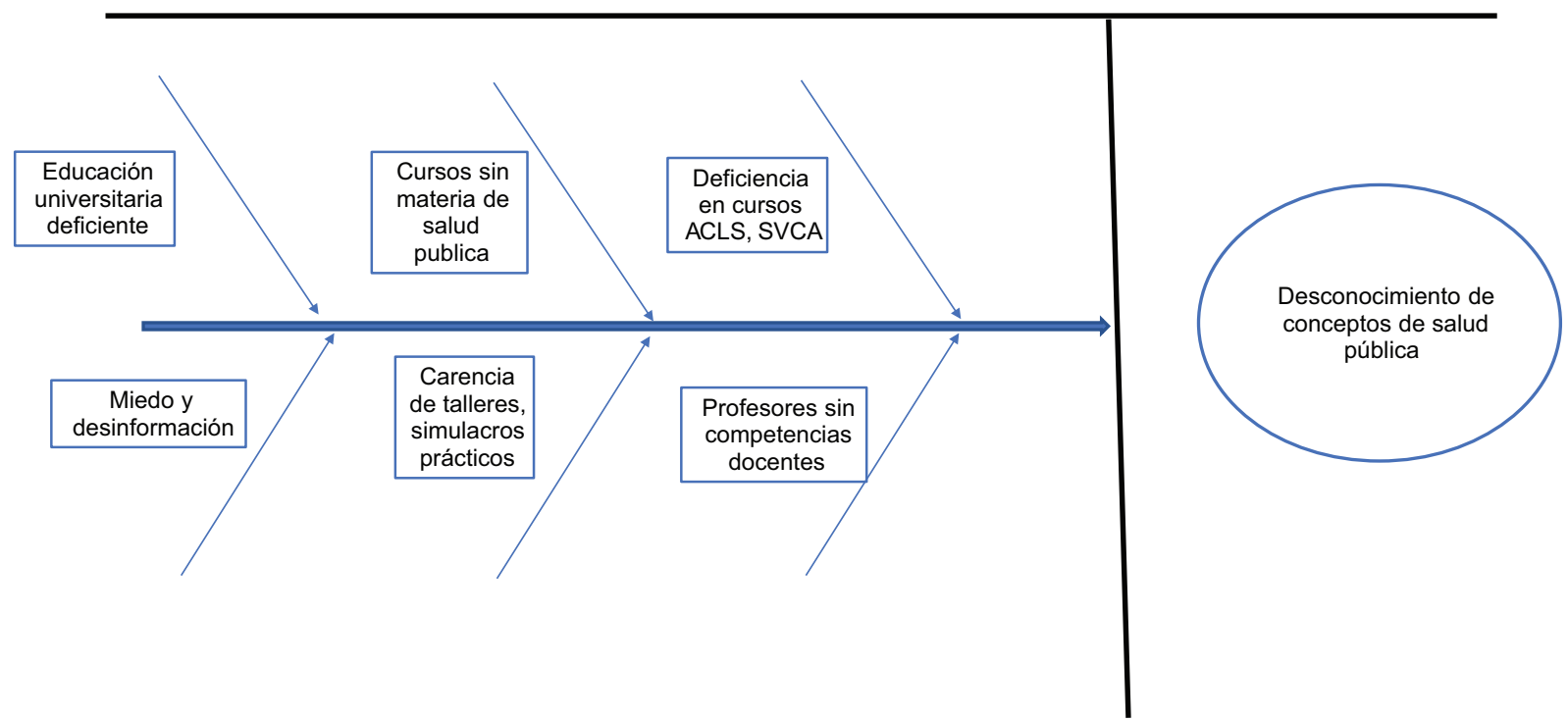

Figura 4. Causas del desconocimiento en materia de salud pública durante una pandemia en profesionales de la salud. $A C L S=A d v a n c e d$ Cardiovascular Life Support, SVCA = Soporte Vital Cardiovascular Avanzad.

experiencia docente y las capacidades de comunicación de sus profesores, además de exigirles mejorar su proceso de enseñanza ${ }^{30}$ (Figura 4).

\section{Enseñanza de manejo de pandemias y desastres}

El documento de preparación y respuesta ante una pandemia elaborado por la Organización Mundial de la Salud establece que la planificación y los preparativos anticipados son fundamentales para atenuar el impacto de las pandemias de influenza. ${ }^{26,31}$

A pesar de lo anterior, la pandemia por el virus de influenza A (H1N1) de 2009 no representó la creación de un programa de largo alcance para los profesionales de la salud; si bien mejoró el sistema de vigilancia epidemiológica, no así la capacidad de respuesta de las instituciones, los hospitales y el personal de salud directamente involucrados en la atención. El escenario ideal sería que cada actor sepa lo que tiene que hacer, cómo y por qué. . $^{9,32,33}$ Tampoco constituyó una mejora en los currículos universitarios para incrementar los conocimientos en materia de salud pública y medicina de desastres. Desafortunadamente, los estudiantes asumirán importantes tomas de decisiones respecto a eventos futuros sin la preparación necesaria, ${ }^{34,35}$ por ejemplo: los anestesiólogos y los especialistas en medicina crítica proporcionan atención médica integral en los servicios de urgencias, quirófanos y unidades de cuidados intensivos, a pesar de lo cual pocos reciben capacitación en medicina de desastres y contingencias epidemiológicas. ${ }^{36}$

La residencia médica representa una oportunidad para implementar intervenciones de capacitación que promuevan comportamientos y habilidades profesionales apropiadas. ${ }^{16}$ La educación sanitaria es necesaria para concientizar a los trabajadores de la salud, ${ }^{37}$ de ahí que se han formulado propuestas para que exista mayor inversión en su formación y puedan ayudar a mitigar los riesgos que plantean los brotes de enfermedades. ${ }^{38}$ La tarea no siempre es sencilla pues existen barreras tecnológicas, de idioma, tiempo y, sobre todo, vinculación a los planes de estudio existentes, cuyos contenidos están saturados (Figura 5). La adquisición de conocimientos basados en simulación se puede encontrar prácticamente en todos los niveles de la capacitación médica; la inversión puede ser alta y requiere una evaluación económica para saber si un sistema es capaz de incluirla en sus programas. ${ }^{39,40}$

Aunado a lo anterior, son pocas las especialidades en las se exigen cursos como Apoyo Vital Avanzado en Trauma, Soporte Vital Básico o módulos encaminados a revisar conceptos de salud pública. Si, además, se suma la falta de talleres y simulacros en el manejo de desastres y de contingencias sanitarias, se explica por qué los estudiantes de medicina y alumnos de las especialidades no 


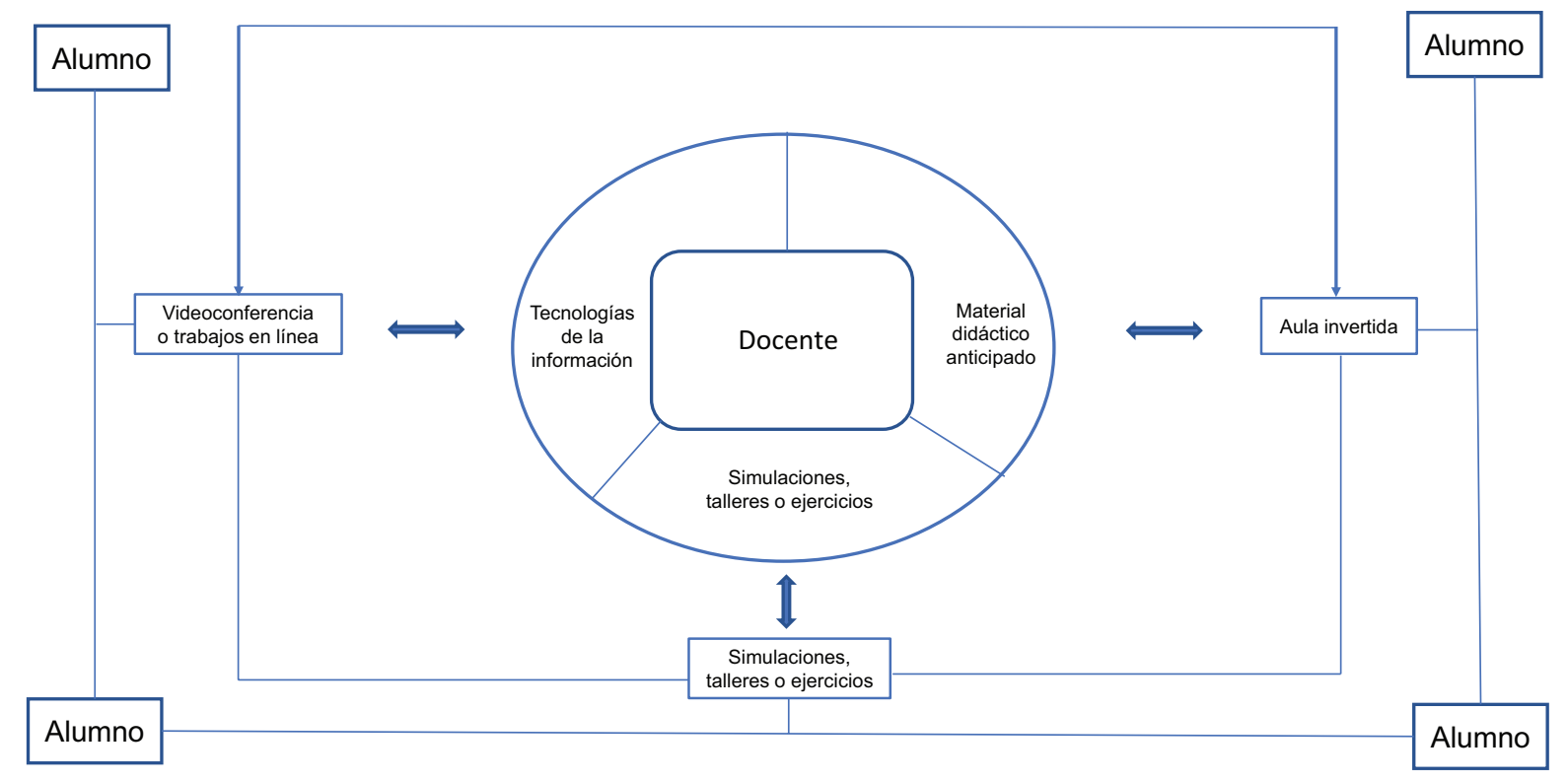

Figura 5. Propuesta de estructura de los cursos durante una pandemia.

pueden ser considerados un grupo de respuesta ante las contingencias sanitarias. ${ }^{41-43}$

\section{Perspectivas y conclusiones}

Los expertos en salud pública han señalado la necesidad de la preparación para enfrentar una pandemia de dimensiones similares a la ocasionada por la influenza en 1918, con el principal problema de no saber cómo ni cuándo se presentará. ${ }^{12}$ Han supuesto que los virus de la gripe subtipo H9 (principalmente H9N2) pueden ser los responsables, debido a sus altas tasas de infección zoonótica y potencial pandémico, razón por la cual han concentrado los esfuerzos en ellos. ${ }^{44}$

A 11 años de la pandemia por el virus de la influenza A (H1N1), emerge el virus SARS-COV-2, con una alta capacidad de trasmisión de persona a persona y una letalidad calculada entre 5 y $6 \%$, el cual ha obligado al aislamiento social, con el consecuente impacto en la economía de todo el mundo. ${ }^{45}$ Las medidas sanitarias aprendidas través de la historia, muchas de ellas desde 1918, un siglo después se implementan en un mundo poco preparado para una crisis sanitaria como la que está ocasionando COVID-19. ${ }^{46}$ Además, se suma el miedo y los problemas de orden psicológico-psiquiátrico, así como la desconfianza del personal de salud respecto a las autoridades, relacionada con el desconocimiento de las medidas de contención y mitigación; quien no conoce los elementos básicos se sentirá amenazado, sin la guía y dirección adecuadas de un líder. ${ }^{47,48}$ La epidemia exterioriza las deficiencias personales e institucionales habituales o "endémicas"; afortunadamente, pueden solventarse mediante inversión en la educación, entre otras cosas. ${ }^{49}$

Contrario a la percepción general de que los médicos son profesionales de respuesta rápida ante desastres y pandemias, las conclusiones plasmadas en diferentes revistas científicas indican que la preparación de los profesionales de la medicina no les permite erigirse como personal que puede enfrentarlos, a pesar de la voluntad para hacerlo. ${ }^{50}$ Más aún, en las crisis existe el riesgo inminente de suspender los cursos académicos, con la consecuente preparación deficiente de los estudiantes, lo que obliga a revisar el proceso de enseñanza.

Las modificaciones estructurales de un programa académico son difíciles debido a todos los factores que influyen, además, dependen de que la organización examine su situación educativa, proponga soluciones, revolucione su sistema y tome en cuenta el mejor escenario para lograr sus objetivos. Tanto las crisis sanitarias como los desastres favorecen el cambio de paradigmas educativos, siempre y cuando no se deje perder la oportunidad.

\section{Conflicto de intereses}

Los autores declaran que no existe conflicto de intereses, ni relación financiera alguna que pueda influir en el contenido de este artículo. 


\section{Financiamiento}

No se ha recibido financiamiento para la realización del manuscrito.

\section{Responsabilidades éticas}

Protección de personas y animales. Los autores declaran que para esta investigación no se han realizado experimentos en seres humanos ni en animales.

Confidencialidad de los datos. Los autores declaran que en este artículo no aparecen datos de pacientes.

Derecho a la privacidad y consentimiento infor-

mado. Los autores declaran que en este artículo no aparecen datos de pacientes.

\section{Bibliografía}

1. Dodd CN, Romio SA, Black S, Vellozzi C, Andrews N, Zuber P, et al. International collaboration to assess the risk of Guillain Barré syndrome following influenza A (H1N1) 2009 monovalent vaccines. Vaccine. 2013;31:4448-4458.

2. Charu V, Chowell G, Palacio-Mejía LS, Echeverría-Zuno S, Borja-Aburto VH, Simonsen L, et al. Mortality burden of the $\mathrm{A} / \mathrm{H} 1 \mathrm{~N} 1$ pandemic in Mexico: A comparison of deaths and years of life lost to seasonal influenza. Clin Infect Dis. 2011;53:985-993.

3. Wong JY, Kelly H, Ip DK, Wu JT, Leung GM, Cowling BJ. Case fatality risk of influenza A (H1N1pdm09): A systematic review. Epidemiology. 2013;24:830-841.

4. Morse SS. Factors in the emergence of infectious diseases. Emerg Infect Dis. 1995:1:7-15.

5. Preparación y respuesta ante una pandemia de influenza. Documento de orientación de la OMS. Suiza: Organización Mundial de la Salud; 2009.

6. Fineberg HV. Pandemic preparedness and response - Lessons from the H1N1 influenza of 2009. N Engl J Med. 2014;370:1335-1342.

7. Bell DM, Weisfuse IB, Hernández-Ávila M, del Río C, Bustamante X, Rodier G. Pandemic influenza as 21st century urban public health crisis. Emerg Infect Dis. 2009;15:1963-1969.

8. Seale H, Leask J, Po K, Maclntyre CR. "Will they just pack up and leave?" - Attitudes and intended behavior of hospital health care workers during an-influenza pandemic. BMC Health Serv Res. 2009;9:30.

9. Aiello A, Khayeri MY, Raja S, Peladeau N, Romano D, Leszcz M, et al. Resilience training for hospital workers in anticipation of an influenza pandemic. J Contin Educ Health Prof. 2011:31:15-20.

10. Gates B. Innovation for pandemics. N Engl J Med. 2018;378:2057-2060.

11. Alpuche-Aranda CM. Infecciones emergentes, el gran reto de la salud global: Covid-19. Salud Pub Mex. 2020;62:123-124.

12. Gates B. Responding to Covid-19, a once in a century pandemic? New Engl J Med. 2020.

13. Lazcano-Ponce $\mathrm{E}$, Alpuche-Aranda C. Alfabetización en salud pública ante la emergencia de la pandemia por Covid-19. Salud Publica Mex. 2020.

14. Lifshitz A. Tendencias de la educación médica. Gac Med Mex. 2000;1:25-33.

15. Hutchins SS, Jansen HA, Robertson SE, Evans P, Kim-Farley RJ. Studies of missed opportunities for immunization in developing and industrialized countries. Bull World Health Organ. 1993;71:549-560.

16. Boyce MR, Katz R. Community health workers and pandemic preparedness: Current and prospective roles. Front Public Health. 2019;7:1-5.

17. Emanuel EJ. Reforming American medical education. Milbank Q. 2017;95:692-697

18. Qualls N, Levitt A, Kanade N, Wright-Jegede N, Dopson S, Reed C, et al. Community Mitigation Guidelines to Prevent Pandemic Influenza - United States, 2017. MMWR. 2017;66:1-34

19. Sambala EZ, Manderson L. Anticipation and response: pandemic influenza in Malawi, 2009. Glob Health Action. 2017;10:1341225.

20. Simonsen L, Chowell G, Andreasen V, et al. A review of the 1918 herald pandemic wave: importance for contemporary pandemic response strategies. Ann Epidemiol. 2018;28:281-288.

21. Rewar S, Mirdha D, Rewar P. Treatment and prevention of pandemic H1N1 influenza. Ann Glob Health. 2015;81:645-653.

22. Sullivan GM. The tragedy of the medical education commons. J Grad Med Educ. 2016;8:1-4.

23. Another step in higher medical education. JAMA. 2016;316:2280.
24. Echevarría-Zuno S, Monroy-Ramírez LE, Palacios-Jiménez NM, Loría-Castellanos SJ, Gallardo-Candelas SA. Estrategia educativa en línea durante el brote de influenza A H1N1. Rev Med Inst Mex Seguro Soc. 2010;48:383-392.

25. Khamis N, Aljumaiah R, Alhumaid A, Alraheem H, Alkadi D, Koppel C, et al. Undergraduate medical students' perspectives of skills, uses and preferences of information technology in medical education: a cross-sectional study in a Saudi Medical College. Med Teach. 2018;40:S68-S76.

26. Hays R. Establishing a new medical school: A contemporary approach to personalizing medical education. Med Teach. 2018;40:990-995.

27. Hiromoto $\mathrm{Y}$, Parchariyanon S, Ketusing N, et al. Isolation of the pandemic (H1N1) 2009 virus and its reassortant with an H3N2 swine influenza virus from healthy weaning pigs in Thailand in 2011. Virus Res. 2012;169:175-181.

28. Kanehira K, Takemae N, Uchida $\mathrm{Y}$, Hikono $\mathrm{H}$, Saito $\mathrm{T}$. Reassortan swine influenza viruses isolated in Japan contain genes from pandemic A(H1N1) 2009. Microbiol Immunol. 2014;58:327-341.

29. Walsh K. Medical education: return on investment. Ulster Med J. 2015;84:111-112.

30. Sandars JE. Critical theory and the scholarship of medical education. Int J Med Educ. 2016;7:246-247.

31. Seale H, Leask J, Po K, Maclntyre CR "Will they just pack up and leave?" - attitudes and intended behavior of hospital health care workers during an influenza pandemic. BMC Health Serv Res. 2009;13.

32. Sočan M, Erčulj V, Lajovic J. Knowledge and attitudes on pandemic and seasonal influenza vaccination among Slovenian physicians and dentists. Eur J Public Health. 2013;23:92-97.

33. Hines L, Rees E, Pavelchak N. Respiratory protection policies and practices among the health care workforce exposed to influenza in New York State: Evaluating emergency preparedness for the next pandemic. Am J Infect Control. 2014;42:240-245.

34. Fink BN, Rega PP, Sexton ME, Wishner C. START versus SALT Triage: Which is preferred by the 21st century health care student? Prehosp Disaster Med. 2018;33:381-386.

35. Costantino C, Amodio E, Calamusa, G, Vitale F, Mazzucco W. Could university training and a proactive attitude of coworkers be associated with influenza vaccination compliance? A multicentre survey among Italian medical residents. BMC Med Educ. 2016;16:38.

36. Tuohetamu S, Pang M, Nuer X, Mohemaiti P, Qin Y, Peng Z, Zheng J, Yu H, Feng L, Feng L. The knowledge, attitudes and practices on influenza among medical college students in Northwest China. Hum Vaccin Immunother. 2017:13:1688-1692.

37. Walsh K, Roma E, Hilton A. Education and support for healthcare professionals to prevent future pandemics: Report of a conference workshop. Ulster Med J. 2018;87:194-196.

38. Hayanga HK, Barnett DJ, Shallow NR, Roberts M, Thompson CB Bentov I, Demiralp G, Winters BD, Schwengel DA. Anesthesiologists and disaster medicine: $A$ needs assessment for education and training and reported willingness to respond. Anesth Analg. 2017:124:1662-1669.

39. Ling Y, Cheng A, Hecker K, Grant V, Currie GR. Implementing economic evaluation in simulation-based medical education: challenges and opportunities. Med Educ. 2018;52:150-160.

40. Robinson LA, Turner IJ, Sweet MJ. The use of gamification in the teaching of disease epidemics and pandemics. FEMS Microbiol Lett. 2018;365:fny111.

41. Pérez-Gómez HR, Ramos-Zúñiga R. La educación médica y su impacto en la salud pública. Perspectivas y retos. Cir Cir. 2012;80:200-208.

42. Mortelmans LJ, Bouman SJ, Gaakeer MI, Dieltiens G, Anseeuw K, Sabbe MB. Dutch senior medical students and disaster medicine: a national survey. Int J Emerg Med. 2015;8:77.

43. Pfeiffer JK. Is the debate and "pause" on experiments that alter pathogens with pandemic potential influencing future plans of graduate students and postdoctoral fellows? mBio. 2015;1:e02525.

44. Peacock THP, James J, Sealy JE, Iqbal M. A global perspective on H9N2 Avian influenza virus. Viruses. 2019;11:620

45. Lai CC, Shih TP, Ko WC, Tang HJ, Hsueh PR. Severe acute respiratory syndrome coronavirus 2 (SARS-CoV-2) and coronavirus disease-2019 (COVID-19): The epidemic and the challenges. Int J Antimicrob Agents. 2020;55:105924.

46. Ren SY, Gao RD, Chen YL. Fear can be more harmful than the severe acute respiratory syndrome coronavirus 2 in controlling the corona virus disease 2019 epidemic. World J Clin Cases. 2020;8:652-657.

47. Huang Y, Zhao N. Generalized anxiety disorder, depressive symptoms and sleep quality during COVID-19 outbreak in China: A web-based cross-sectional survey. Psychiatry Res. 2020;288:112954.

48. Shi Y, Wang J, Yang Y, Wang Z, Wang G, Hashimoto K, et al. Knowledge and attitudes of medical staff in Chinese psychiatric hospitals regarding COVID-19. Brain Behav Immun Health. 2020;4:100064.

49. Gates B. The next epidemic- Lessons from Ebola. N Engl J Med. 2015;372:1381-1384.

50. Tomášková H, Boháčová S, Šlachtová H. Attitudes of the medical students from two Czech universities to pandemic Flu A (H1N1) 2009 and to influenza vaccination. Cent Eur J Public Health. 2012;20:215-218. 